\title{
Etude Sedimentologique Et Stratigraphique Du Remplissage Du Grand Porche Du Gisement De Montgaudier, Charente, France
}

\author{
Boukhir Mohammed, (PH) \\ Chaouni Abdel-Ali, (PES) \\ Lahrach Abderrahim, (PES) \\ Laboratoire Géoressources et Environnement \\ Université sidi Mohamed Ben Abdellah, Maroc
}

doi: 10.19044/esj.2017.v13n18p402 URL:http://dx.doi.org/10.19044/esj.2017.v13n18p402

\begin{abstract}
The sedimentological study of the deposits accumulated at the level of the two sectors of the Grand Porche of Montgaudier allowed establishing a stratigraphic synthesis of the deposits in this part of the filling of this deposit and the paleoclimatic conditions of the establishment of the different types of sediments. The systematic analyzes carried out on samples taken from the two sectors I and II revealed their origins: allochthonous and autochthonous. The data from these sedimentological analyzes of this filling also revealed the conditions and the factors which led to the establishment of these deposits. Finally, these stratigraphic studies allowed establishing a chronological framework relative to the chronology of the deposits of the adjacent loci, 8 and 6, attributed to the old würm and the Riss-würm.
\end{abstract}

Keywords: Grand porche, Sedimentology, granulometry, correlation, stratigraphy, morphoscopy, concretions, cold climate, temperate and humid climate, chronostratigraphy, würm, Charente, France

\section{Résumé}

L'étude sédimentologique des dépôts accumulés au niveau des deux secteurs du Grand Porche de Montgaudier a permis d'établir une synthèse stratigraphique des dépôts dans cette partie du remplissage de ce gisement et les conditions paléoclimatiques de la mise en place des différents types de sédiments. Les analyses systématiques effectués sur les échantillons prélevés des deux secteurs I et II ont permis de connaître leurs origines : allochtone et autochtone. Les données de ces analyses sédimentologiques de ce remplissage ont révélé également les conditions et les facteurs qui ont présidé à la mise en place de ces dépôts. Enfin, ces études stratigraphiques 
ont permis d'établir un cadre chronologique relatif par rapport à la chronologie des dépôts des locus adjacents, en l'occurrence 8 et 6 , attribués au würm ancien et au Riss-würm.

Mots clés : Grand porche, Sédimentologique, granulométrie, corrélation, stratigraphie, morphoscopie, concrétions, climat froid, climat tempéré et humide, chronostratigraphie, würm, Charente, France

\section{Introduction}

Le gisement de Montgaudier est l'un des plus importants sites paléolithiques de Charente. Son étude sédimentologique demeure du plus haut intérêt, afin de mieux connaitre sa lithostratigraphie global et d'essayer de le situer dans le cadre chronologique du Quaternaire charentais.

Etant donné la grandeur et la complexité topographique de ce gisement, et pour mieux comprendre cette complexité de son remplissage, nous avons séparé cette recherche en cinq parties distinctes:

- une première partie est réservée à l'étude stratigraphique et sédimentologique du Grand porche qui regroupe les loci 1, 2, 3 et 5 .

- Une deuxième partie sera consacrée à l'étude du premier étage ou locus 6 .

- La troisième partie regroupera l'étude de l'abri Gaudry ou locus 8 et celle de l'abri Lartet.

- La quatrième partie traitera les locus 2, locus 12 et locus 11.

- La cinquième partie abordera l'étude des minéraux lourds et légers de la fraction sableuse ainsi que l'analyse des minéraux argileux d'échantillons prélevés dans différents secteurs de ce gisement.

Le présent travail comportera les résultats de recherches effectuées uniquement sur la partie Grand porche.

\section{Situation géographique du gisement de Montgaudier (fig.1).}

Le gisement de Montgaudier se situe à $2 \mathrm{~km}$ au Nord ouest de la commune de Montbron à une vingtaine de kilomètres de la ville d'Angoulème, département de Charente. De coordonnées géographiques $45^{\circ} 41^{\prime}$ latitude Nord, $0^{\circ} 28^{\prime}$ longitude Est, Cette grotte s'ouvre dans les calcaires dolomitiques bajociens à une altitude de près de $100 \mathrm{~m}$ altitude. Il se localise sur la rive droite de la Tardoire, affluent de la Charente, France. Elle est composée de plusieurs loci et abris plus ou moins conservés dont les sédiments de remplissage montent une stratigraphie très complexe (Boukhir, 1992 ; 2017 ; Debenath, 1974). 


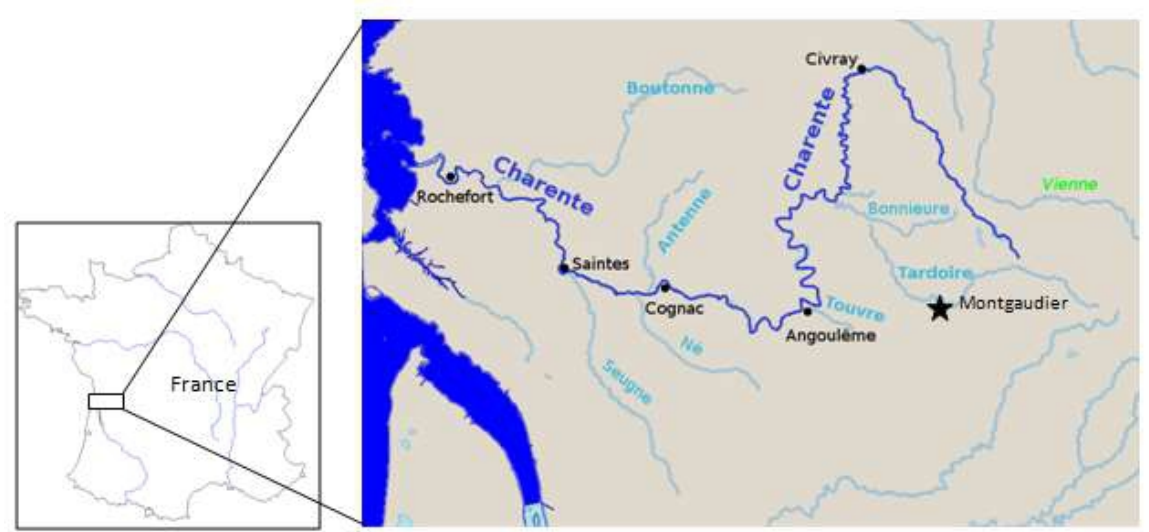

Fig.1 Situation géographique du gisement de Montgaudier

Grand porche, secteur I ou locus 5 (Fig. 2).

Situation des coupes étudiées (Fig. 3).

Le secteur I est constitué par le locus 5. Deux profils 1 et 2 ont été réalisés dans se secteur Deux séries d'échantillons ont été ainsi prélevées au niveau de chacun de ces deux profils.

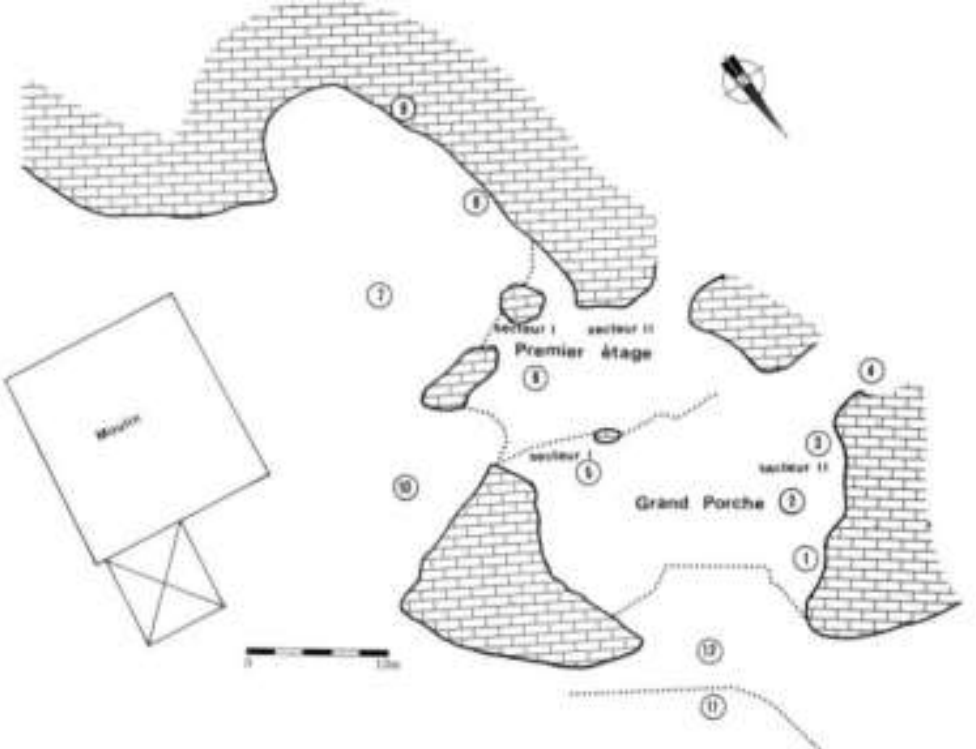

Fig. 2 : Plan général de Montgaudier : situation des secteurs 1 et 2 au premier étage. 


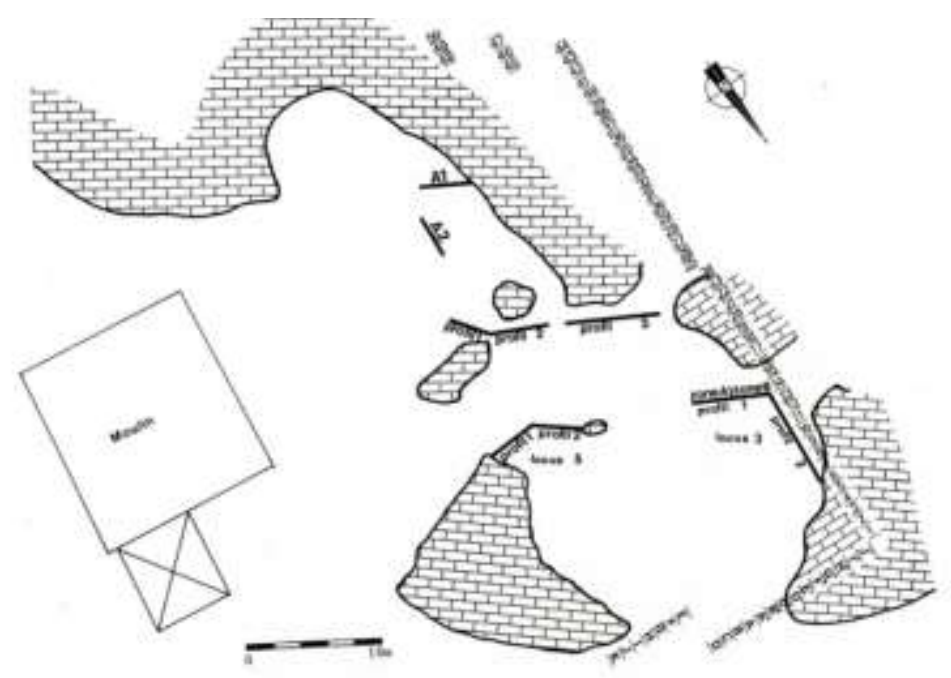

Fig. 3 : Localisation des différents profils réalisés au Grand porche.

Stratigraphie.

Grand porche, secteur I, profil 1 (fig.4)

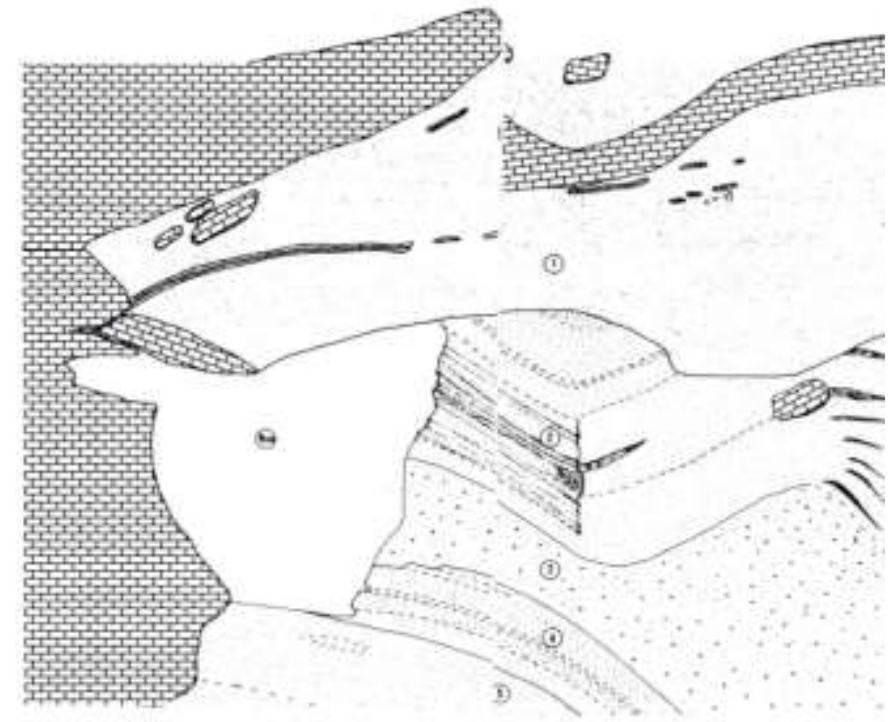

Fig. 4 : Coupe stratigraphique Grand porche, secteur I : profil 1.

Dans cette zone, on distingue de bas en haut les unités stratigraphiques suivantes: 
Couche 5 : elle est observée sur une épaisseur de $45 \mathrm{~cm}$ au maximum. Sa base n'a pas été atteinte. Elle est composée de sables siliceux, emballant quelques rares granules quartzitiques de petites dimensions.

Couche 4: d'épaisseur maximale $35 \mathrm{~cm}$, elle est formée par un ensemble de niveaux sableux très concrétionnés, intercalés avec des niveaux de sables siliceux peu consolidés. Ces sables renferment quelques granules de quartz et de quartzites de petites dimensions.

Couche 3: d'épaisseur maximale $1 \mathrm{~m}$, il s'agit d'un dépôt de sables chargés en granules et graviers de quartz et de quartzites. Ces éléments grossiers sont assez émousses dans leur quasi totalité. Le sédiment de cette couche est peu consolidé.

Couche 2: d'épaisseur maximale $80 \mathrm{~cm}$, cette couche regroupe plusieurs niveaux de sables siliceux, lités et très consolidés dans la partie Est de la coupe. Ces niveaux sont alternés par des passages sableux peu compacts. Ce litage disparait vers l'ouest de cette zone où des taches d'oxydes de manganèse font leur apparition sous forme de petites lentilles allongées.

Couche 1bis: il s'agit d'un dépôt sablo-limoneux. Stratigraphiquement, ce dépôt semble postérieur aux couches 5 à 2 .

Couche 1: constituée de dépôt formé de sable siliceux, renfermant quelques granules quartzeux. Elle repose en discordance sur toutes les couches sous-jacentes.

\section{Grand porche, secteur I, profil 2 (fig. 5)}

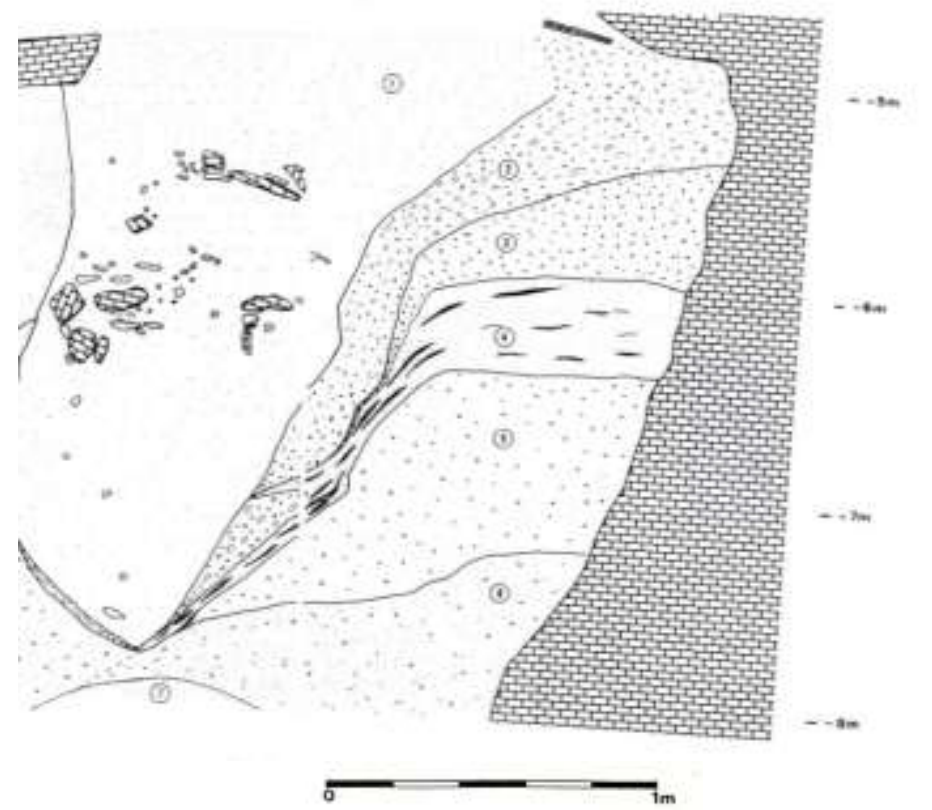

Fig. 5 : Coupe stratigraphique Grand Porche, secteur I : profil 2. 
Ce profil s'observe sur une puissance de 3,50 m environ. Dans cette coupe, les dépôts se divisent en deux parties bien distinctes :

- une partie inférieure regroupant les couches 7 à 2, composée de sédiments, d'origines allochtones non calcaires;

- une partie supérieure comprenant uniquement la couche 1. Son dépôt est en grande majorité d'origine autochtone et calcaire. Dans le détail des caractéristiques lithostratigraphiques, on observe de bas en haut les unités suivantes :

Couche 7 : sa base n'a pas été atteinte. Elle est toutefois visible sur une épaisseur de $10 \mathrm{~cm}$ et environ un mètre de long. Elle est composée de sables siliceux contenant quelques granules quartzeux dispersés dans une matrice de couleur grise. Le sédiment est très peu compact.

Couche 6 : elle est visible sur une épaisseur de $80 \mathrm{~cm}$ au maximum. Elle s'amenuise progressivement en allant vers 1'Est de la coupe pour disparaitre pratiquement en biseau. Il s'agit d'un sédiment sableux faiblement limono-argileux, emballant des granules siliceux et quelques rares petits galets de roches cristallines. Le sédiment fin est de couleur jaune. De structure particulaire et peu compact.

Couche 5: d'épaisseur maximale un mètre. Elle est horizontale à l'Ouest de la coupe, faiblement inclinée vers sa partie Est où elle a été pratiquement tronquée par les dépôts de la couche 1. Le sédiment est formé essentiellement de sables quartzeux, riche en granules siliceux, peu compact et friable. Le matériel fin est de couleur brun foncé. Cette couche renferme également des traces d'oxydes de fer et de manganèse.

Couche 4 : d'épaisseur maximale $50 \mathrm{~cm}$, elle est constituée de sables siliceux. Elle est horizontale dans la partie Ouest de la coupe, plonge vers l'Est tout en ravinant partiellement la couche sous-jacente. Le dépôt de cette couche s'injecte légèrement dans la couche 2 avant qu'il ne disparaisse en biseau en allant vers l'Est du profil. Le sédiment est peu compact et de couleur grise jaunâtre. Au sein de cette couche se développent également des taches de sels de manganèse sous forme de lentilles allongées, peu épaisses et à contours diffus.

Couche 3 : d'épaisseur maximale $60 \mathrm{~cm}$, cette couche n'apparait que dans la partie Ouest de la coupe. Elle est composée essentiellement de galets et de graviers de roches cristallines, enrobés dans une matrice de sables siliceux. Elle est horizontale dans sa partie Ouest. Elle forme une coulée assez mince vers l'Est où elle se termine en biseau. Les sédiments de cette couche sont non structurés et peu compacts et de couleur grise.

Couche 2 : d'épaisseur maximale $55 \mathrm{~cm}$, le dépôt de cette couche est de texture nettement plus grossière que celle de la couche sous-jacente. Il s'agit d'un dépôt formé essentiellement de sables siliceux très chargés en 
galets et graviers. Les galets sont de nature pétrographique assez variée, rappelant celle des galets provenant du locus 3. Ils sont composés généralement de quartz et de quartzites, associés à des galets de roches granitiques, gréseuses et de silex. Les graviers et les granules sont formés exclusivement de quartz et de quartzites. Le dépôt de cette couche est peu compact. Le sédiment fin est de couleur brun grisâtre. Cette couche est aspirée en grande partie dans la région médiane de cette zone et entre directement en contact avec la couche 4 qui est à son tour fortement aspirée. Elle disparait ensuite sous les dépôts de la couche 1.

Couche 1 : elle est constituée d'une matrice sablo-argilo-limoneuse emballant des cailloutis carbonatés de taille variable. Elle ravine en grande partie les dépôts sous-jacents sur une profondeur de 2,40 $\mathrm{m}$. Le dépôt de cette couche est peu compact. Le sédiment fin est de couleur jaune. La séquence se termine par un plancher stalagmitique épais de 5 à $6 \mathrm{~cm}$ dont des lambeaux sont visibles sur une longueur de $30 \mathrm{~cm}$ environ. Ce plancher correspond certainement au plancher stalagmitique qui apparait au sommet du profil 1 sur plus de 1,30 m. Du point de vue stratigraphique, ce plancher semble antérieur aux dépôts qui forment la couche 1 dans chacune des deux zones de ce locus. Cependant, il a été vraisemblablement tronqué par les sédiments qui ont glissé par-dessus lors de l'aspiration karstique qui a affecté cette partie du remplissage.

\section{Etude sédimentologique du profil 1, secteur I Diagramme stratigraphique global}

Ce diagramme fait apparaître des variations sensibles entre les différentes unités stratigraphiques de cette partie du remplissage. Les éléments de diamètre supérieur à $10 \mathrm{~mm}$ sont pratiquement absents dans les couches 5,4 et 2 . Ils apparaissent timidement dans les couches 3 et 1 où ils constituent respectivement 3 et $1 \%$ du sédiment total. La fraction fine entre pour 5 et $7 \%$ dans la composition des dépôts des couches 5 et 4 . Elle est bien développée dans la couche 3 où elle forme $28 \%$ de son sédiment global. Elle constitue près de $13 \%$ de l'ensemble du sédiment.

\section{Morphoscopie des grains de quartz}

L'examen morphoscopique des grains de quartz révèle la dominance des sub-anguleux luisants. Cette catégorie de grains constitue près de $70 \%$ dans la couche 5 . Leur proportion augmente ensuite jusqu'au niveau de couche 3 pour former $80 \%$ du cortège quartzeux, puis diminue en allant vers le sommet de ce remplissage où leur pourcentage atteint tout de même $60 \%$. Les grains émoussés luisants sont peu abondants; ils entrent pour 8 à $14 \%$ dans la composition du cortège analysé. Leur taux maximum s'enregistre dans la couche 3 . Les grains non usés sont relativement plus fréquents que 
les émoussés luisants, sauf dans la couche 3 où ils forment à peine $6 \%$. Dans les autres couches, ils entrent pour 14 à $20 \%$ dans la composition des grains de quartz. Enfin, aucun grain d'aspect mat n'a été observé dans ces sédiments.

\section{Etude sédimentologique du profil 2 , secteur I Diagramme stratigraphique global}

Ce diagramme permet de mettre en évidence quatre ensembles sédimentaires :

Ensemble 1 : il regroupe les couches 7 à 5. Dans ces couches, les fractions de diamètre supérieur à $2 \mathrm{~mm}$ sont toujours présentes. Les galets qui la composent sont de petites dimensions et ne dépassent guère $3 \mathrm{~cm}$ de diamètre. Les éléments grossiers sont composés essentiellement de quartz et quartzites, associés à de rares petits galets de roches granitiques et gréseuses. Morphoscopiquement, les galets sont émoussés à très émoussés. Dans la couche 7, les granules portent des traces d'oxyde de manganèse. A ces éléments siliceux, viennent s'ajouter quelques rares petites concrétions ferrugineuses dans la couche 6.

Ensemble 2 : représenté uniquement par la couche 4 , cet ensemble s'individualise par un appauvrissement en éléments de diamètre supérieur à 2 $\mathrm{mm}$. Les éléments grossiers ne dépassent pas $5 \mathrm{~mm}$ de diamètre et forment seulement $5 \%$ du sédiment global.

Ensemble 3 : il englobe les couches 3 et 2 et se caractérise par un enrichissement en éléments grossiers. Ces derniers représentent près de $15 \%$ du dépôt dans la couche 2. Elle entre pour $47 \%$ dans la composition du dépôt de la couche 3 et pour $24 \%$ du sédiment provenant de la couche 2 .

Ensemble 4 : il correspond à la couche 1. Dans cet ensemble, les éléments grossiers de diamètre compris entre $2 \mathrm{~mm}$ et $10 \mathrm{~cm}$ forment près de $16 \%$ du dépôt de cette couche. Les éléments grossiers sont composés essentiellement de cailloutis calcaires, associés à quelques galets et granules hérités des dépôts sous jacents par remaniement.

\section{Morphoscopie des grains de quartz}

Les grains sub-anguleux luisants sont largement dominants dans tous les échantillons. Ce type de grains forme plus de $80 \%$ du cortège de quartz, sauf dans la couche 1 où leur proportion ne dépasse pas $60 \%$. Les émoussés luisants sont moins nombreux mais constituent tout de même près de $10 \%$ de l'ensemble des grains examinés. Les sub-anguleux à peine mats n'apparaissent qu'au niveau de la couche 1. Les non usés sont relativement peu abondants. Ils perdent progressivement de leur valeur de la couche 7 (25 $\%$ ) à la couche $2(8 \%)$. 


\section{Interprétation dynamique et climatique des dépôts}

Les observations effectuées sur le terrain ainsi que les données des analyses granulométriques des deux séries d'échantillons prélevés dans cette partie du remplissage montrent que les dépôts n'évoluent pas de la même façon dans les deux profils. A l'exception de la couche 1, profil 2, les sédiments sont d'origine allochtone, puisqu'ils sont composés essentiellement d'éléments de roches cristallines, alors que la roche encaissante est exclusivement carbonatée et de texture très fine. Plusieurs arguments témoignent que ces sédiments ont été transportés et mis en place par ruissellements. Le premier indicateur est l'aspect luisant de la quasitotalité des grains de sables quartzeux et cela dans tous les échantillons analysés. D'autre part, la présence d'éléments grossiers (galets et granules) assez émoussés témoigne d'un transport par l'eau. En se basant sur les résultats que nous avons obtenus, on constate alors que les variations assez importantes de texture des dépôts témoignent des variations de l'intensité des ruissellements qui les ont transportés dans cette partie du remplissage. Pour le profil 1, la pauvreté de la couche 5 en éléments à diamètre supérieur à 2 $\mathrm{mm}$ laisse supposer un climat tempéré et modérément humide. Par ailleurs, le bon classement et l'homogénéité des sédiments témoignent d'une mise en place par ruissellement réguliers et peu intenses. Dans la couche 4, les niveaux sableux correspondent vraisemblablement à des épisodes de ruissellements d'intensité modérée, séparés par des épisodes plus secs indiquant des arrêts de ce type de sédimentation. Au niveau de la couche 3, l'abondance relative des éléments grossiers de diamètre supérieur à $2 \mathrm{~mm}$ témoigne d'un climat plus humide que celui qui a présidé la mise en place des sédiments de la couche 5, et par conséquent les ruissellements étaient nettement plus intenses. D'autres part, le mauvais classement des sédiments et l'absence d'un granoclassement de ces matériaux laisse envisager un dépôt par excès de charge. Dans la couche 2, le degré d'humidité diminue à nouveau comme nous l'indique l'absence d'éléments grossiers dans ces sédiments. L'alternance de niveaux concrétionnés avec des niveaux non consolidés de texture identique, peut être interprétée comme une mise en place de sédiments par ruissellement pendant des épisodes relativement humides, suivies de périodes assez sèches qui ont conditionné le concrétionnement des dépôts. En ce qui concerne les dépôts supérieurs de la couche 1, ils sont significatifs d'un climat relativement tempéré et humide. Le caractère bien classé du sédiment de cette couche traduit une mise en place par ruissellement continu et régulier. Enfin, la présence d'un plancher stalagmitique dans cette partie du remplissage, indique des conditions climatiques relativement chaudes et humides. Pour ce qui est du profil 2, la couche 7 témoigne des conditions climatiques semblables à celles qui ont présidé au dépôt de la couche 5, profil 1, si l'on se base sur la comparaison 
des deux séquences stratigraphiques. Cependant, la mise en place de ces deux couches ( 7 du profil 2 et 5 du profil 1) ne semble pas contemporaine puisqu'elles ne présentent pas les mêmes caractéristiques sédimentologiques. Le bon classement du matériel composant la couche 7 montre qu'elle a été déposée par un ruissellement moyennement intense et assez régulier. A partir de la couche 6 , on assiste à une augmentation du degré de l'humidité jusque dans la couche 5 comme en témoigne la présence d'éléments grossiers et de sels ferrugineux. L'humidité perd légèrement de son importance pendant la mise en place des sédiments de la couche 4 . Cette dernière semble être ravinée en partie par les ruissellements qui ont déposés les sédiments de la couche 3 nettement plus chargés en éléments grossiers de diamètre supérieur à $2 \mathrm{~mm}$, ce qui suggère des courants d'eau plus compétents témoignant de période plus d'humide. Les dépôts de la couche 3 riches en éléments grossiers correspondent à un climat encore plus humide et des ruissellements plus compétents susceptibles de transporter et mettre en place des galets pouvant atteindre la taille de $5 \mathrm{~cm}$ d'origine allochtone.

\section{Corrélations stratigraphiques entre le profil 1 et 2 du secteur I}

Les variations latérales entre les deux profils 1 et 2 sont dues soit aux variations texturales et par conséquent aux conditions de mise en place des sédiments, soit à des processus secondaires, postérieurs aux dépôts tels que le concrétionnement des sédiments. A ces processus, il faut ajouter le phénomène de l'aspiration karstique qui a affecté pratiquement tous les dépôts de cette partie du remplissage. Cependant, la couche 2 du profil 1 peut être corrélée avec la couche 4 du profil 2, leurs sédiments ayant des caractéristiques sédimentologiques identiques. Les données granulométriques et les caractéristiques sédimentologiques des sédiments nous permettent également d'établir la correspondance entre la couche $5 \mathrm{du}$ profil 2 et la couche 3 du profil 1.

\section{Grand porche, secteur II ou locus 3} Situation des profils étudiés (Fig. 3)

Le secteur comprend les loci 1, 2 et 3. Deux profits ont été relevés dans se secteur : Un profil 1 est séparée à son tour en deux zones A et B. Un profil 2 orienté vers l'Ouest. 


\section{Grand porche (locus 3), secteur II, profil 1 (fig. 6)}

\section{Profil 1, zone A}

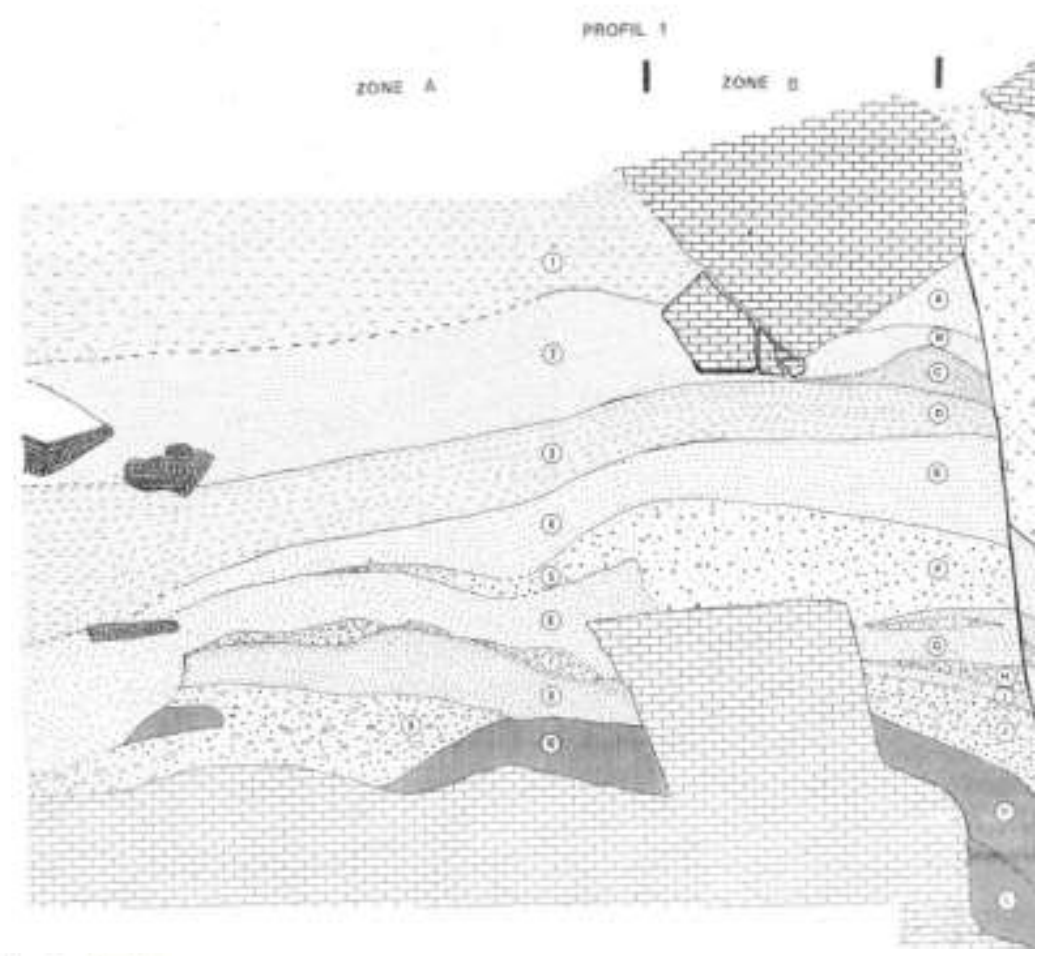

Fig. 6 : Coupe stratigraphique au niveau du Grand Porche : secteur II, profil 1 (zones A et B)

On distingue, de bas en haut, les unités stratigraphiques suivantes :

Couche 10: d'épaisseur maximale $40 \mathrm{~cm}$, cette couche repose directement sur un très puissant bloc calcaire qui sert de substratum à cette formation. Elle a été ravinée en partie avant la mise en place des formations sus-jacentes. Son dépôt est composé d'argiles limoneuses.

Couche 9: d'épaisseur maximale $60 \mathrm{~cm}$, elle est formée de sables siliceux à nombreux granules et galets de roches cristallines notamment. Les galets sont émoussés à sub-émoussés, de taille ne dépassant pas $5 \mathrm{~cm}$ de long.

Couche 8: d'épaisseur maximale $50 \mathrm{~cm}$, elle est constituée essentiellement de limons et d'argiles. Cette couche repose directement sur la couche 9, mais également sur la couche 10 vers la partie Ouest de cette zone.

Couche 7: d'épaisseur maximale $20 \mathrm{~cm}$, elle a été tronquée par la couche supérieure. Son sédiment est formé de granules mais aussi de galets ne dépassant pas $5 \mathrm{~cm}$ de diamètre, le tout emballé dans une matrice 
composée principalement de sables siliceux. Les galets sont émoussés à subémoussés.

Couche 6 : d'épaisseur maximale $35 \mathrm{~cm}$, cette couche est formée de sables limono-argileux de couleur jaunâtre. Le sédiment est peu compact.

Couche 5 : d'épaisseur maximale $70 \mathrm{~cm}$, il s'agit d'un dépôt sableux contenant des granules et galets de nature pétrographique variés. Les galets sont, émoussés à sub-émoussés. Cette couche repose en discordance sur le dépôt sous-jacent et disparait en biseau vers 1'Est du profil.

Couche 4 : d'épaisseur maximale $45 \mathrm{~cm}$, elle est constituée d'un sable argileux. Le sédiment est faiblement consolidé.

Couche 3: d'épaisseur maximale $60 \mathrm{~cm}$, elle est formée de sables argilo- limoneux. Le sédiment est peu compact et de couleur brun jaunâtre.

Couche 2 : d'épaisseur maximale $80 \mathrm{~cm}$, il s'agit d'un dépôt de texture sablo-limono- argileuse et de structure laminaire.

Couche 1: d'épaisseur supérieure à $50 \mathrm{~cm}$, cette couche a été remaniée dans sa partie sommitale. Son sédiment présente une texture limono-argileuse. Il présente des traces d'activités biologiques (terriers). Plancher stalagmitique : il coiffe les dépôts de la couche 2 et se développe sur une épaisseur de $15 \mathrm{~cm}$.

\section{Profil I, zone B}

Cette zone est limitée à l'ouest par une ligne de faille, provoquée par l'affaissement des dépôts du profil 2. Dans cette partie du remplissage, on distingue de bas en haut les unités stratigraphiques suivantes :

Couche L: d'épaisseur maximale $50 \mathrm{~cm}$; cette couche repose directement sur un gros bloc calcaire d'effondrement. Elle est composée d'argiles limoneuses peu sableuses. On note également la présence de taches de couleur brun foncé et à contours diffus.

Couche K : d'épaisseur maximale $60 \mathrm{~cm}$, elle se présente sous forme d'une lentille allongée. Elle perd progressivement de son importance vers l'Ouest de la coupe avant de disparaitre en biseau. Le sédiment qui la compose est un limon argileux peu sableux.

Couche $\mathrm{J}$ : d'épaisseur maximale $45 \mathrm{~cm}$, il s'agit d'un sable siliceux, emballant des granules et des galets pouvant atteindre $7 \mathrm{~cm}$ de diamètre. Les éléments grossiers comprennent essentiellement des fragments de roches cristallines, mais on rencontre également quelques galets gréseux. Les éléments grossiers sont souvent émoussés à sub- émoussés.

Couche 1: d'épaisseur maximale $10 \mathrm{~cm}$, elle est constituée de sables quartzeux englobant quelques rares granules siliceux.

Couche $\mathrm{H}$ : l'épaisseur maximale $20 \mathrm{~cm}$, elle s'amenuise progressivement pour se biseauter vers $1^{\prime} E s t$ du profil. C'est un sable grossier, siliceux, chargé en granules et galets de petites dimensions, ne 
dépassant pas $5 \mathrm{~cm}$ de diamètre. Les éléments grossiers sont de nature pétrographique cristalline. Les galets sont émoussés à sub-émoussés.

Couche G : d'épaisseur maximale $35 \mathrm{~cm}$, elle est composée de sable limono-argileux. Sa limite inférieure est irrégulière.

Couche F: d'épaisseur maximale $70 \mathrm{~cm}$, cette couche est la continuité de la couche 5 décrite dans la zone A. Elle est composée de granules et de galets non calcaires emballés dans une matrice de sables siliceux. Le sédiment est assez friable.

Couche E : d'épaisseur maximale $70 \mathrm{~cm}$, c'est le prolongement de la couche 4 décrite dans la zone $\mathrm{A}$. Le sédiment est très peu consolidé.

Couche D : d'épaisseur maximale $10 \mathrm{~cm}$, elle représente l'extension de la couche 3 de la zone $\mathrm{A}$ et dont les sédiments sont de même nature lithologique dans tout le profil 1.

Couche $\mathrm{C}$ : d'épaisseur maximale $16 \mathrm{~cm}$, elle n'existe qu'au niveau de cette zone du profil 1. Elle se développe sous forme d'une lentille qui se termine en biseau vers l'Est du profil. Son sédiment est composé de sables micacés. On trouve également des traces de manganèse au niveau de cette couche.

Couche B : d'épaisseur maximale $25 \mathrm{~cm}$; il s'agit d'un sable limonoargileux de structure laminaire, assez compact et de couleur jaune.

Couche $\mathrm{A}$ : cette couche est tronquée à son sommet. Elle est constituée de limons argilo- sableux et montre des traces d'activités de terriers. Le sédiment est assez compact. 
Grand porche, secteur II, profil 2 (fig. 7)

Stratigraphie

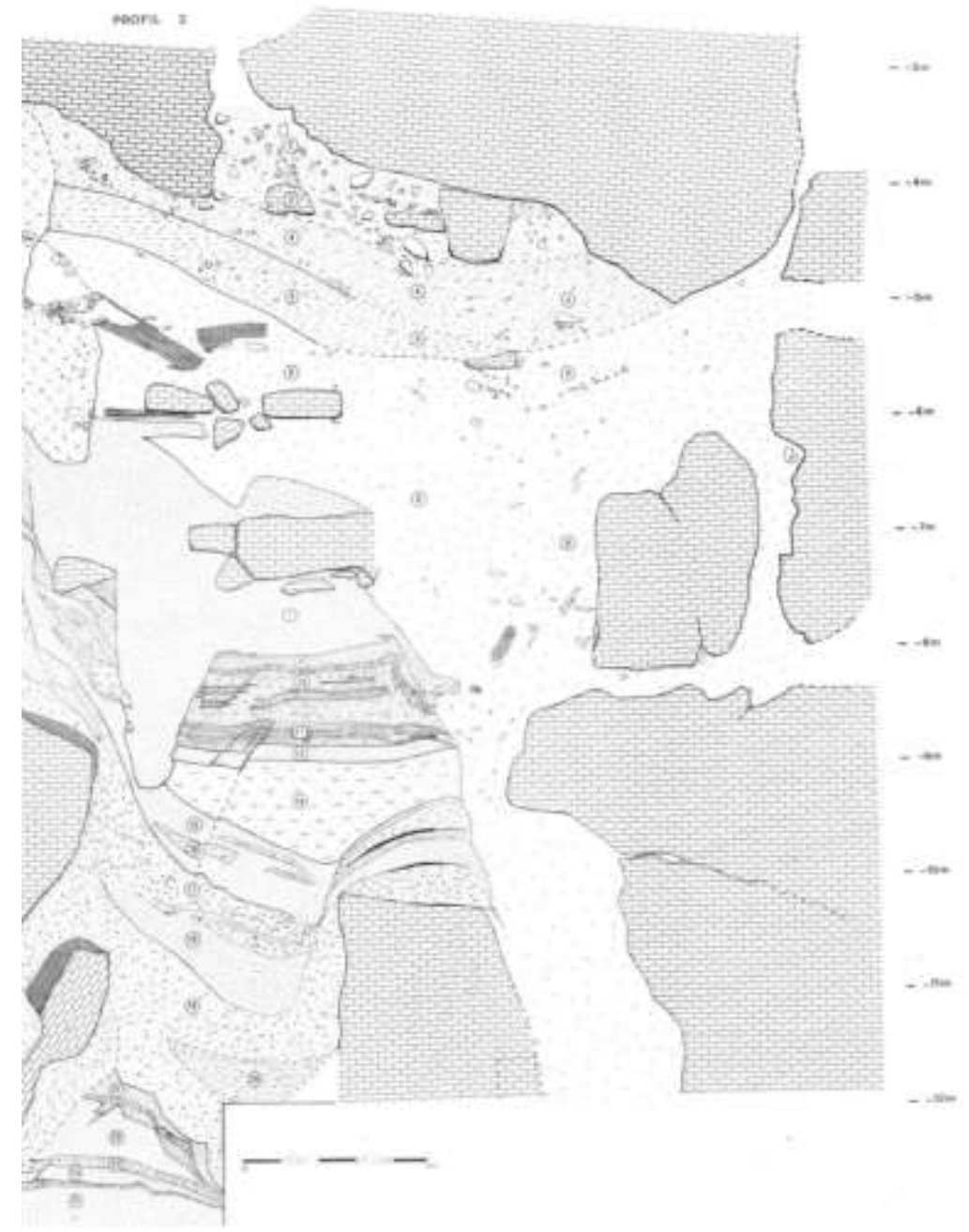

Fig. 7 : Coupe stratigraphique au niveau du Grand Porche : secteur II, profil 2

Dans cette partie du remplissage, on distingue deux types de dépôts formant deux grands ensembles sédimentologiques bien distincts :

Un ensemble inférieur constitué d'un dépôt non calcaire, à caractère fluviatile et d'origine allochtone. Un ensemble supérieur composé de dépôt essentiellement calcaire d'origine autochtone. Dans le détail, on distingue de bas en haut les unités stratigraphiques suivantes :

Ensemble inférieur :

Couche 26 : elle est visible sur une épaisseur maximale de $15 \mathrm{~cm}$. Il $s^{6}$ agit d'un sable argilo-limoneux, peu compact et de couleur jaune pâle. 
Couche 25 : d'épaisseur maximale $6 \mathrm{~cm}$, elle composée de sédiment à éléments grossiers emballés dans une matrice sableuse. Les éléments grossiers sont des galets de quartz, de quartzites plus ou moins altérés et quelques rares fragments gréseux. Les galets sont souvent émoussés à très émoussés.

Couche 24 : d'épaisseur maximale $15 \mathrm{~cm}$, elle est composée d'un sédiment sableux, faiblement argilo-limoneux, de structure laminaire. Le sédiment est peu compact.

Couche 23: d'épaisseur maximale $10 \mathrm{~cm}$. Elle est constituée d'un sable faiblement argilo-limoneux, englobant des granules et des galets de petites dimensions. La nature pétrographique de ces éléments est identique à celle de la couche 25 .

Couche 22: d'épaisseur maximale $50 \mathrm{~cm}$, elle est formée de sables siliceux de structure grenue. Cette couche s'amenuise vers le Nord, pour disparaitre finalement en biseau.

Couche 21 : d'épaisseur maximale $30 \mathrm{~cm}$, le sédiment de cette couche est de texture sable-argilo-limoneuse, peu compact et de couleur grise.

Couche 20: elle est d'épaisseur très variable. Le sédiment qui la constitue est compose de sables siliceux, charges de granules et de graviers. Les éléments grossiers sont essentiellement composés de quartz et des quartzites sub-émoussés à émoussés.

Couche 19: elle est d'épaisseur très variable et elle est formée de sables argilo-limoneux à rares éléments grossiers de nature cristalline et de petites dimensions. Les galets rencontrés dans ce dépôt sont généralement émoussés.

Couche 18: d'épaisseur maximale $50 \mathrm{~cm}$, elle est constituée d'un sable argileux, faiblement limoneux. Le sédiment de cette couche est peu compact.

Couche 17 : d'épaisseur maximale $50 \mathrm{~cm}$, cette couche est composée de sables chargés de galets et de granules. La pétrographie des éléments grossiers est de nature cristalline. La dimension des galets ne dépasse pas 3 $\mathrm{cm}$ de diamètre au maximum. Le dépôt de cette couche se présente sous forme d'une coulée qui recouvre en grande partie les couches sous- jacentes.

Couche 16 : elle se développe sous forme de petites lentilles longues de 30 à $90 \mathrm{~cm}$ et épaisses de 5 à $15 \mathrm{~cm}$. Le sédiment de cette couche est constitué essentiellement de sables renfermant quelques granules de quartz et de quartzites.

Couche 15 : d'épaisseur maximale $60 \mathrm{~cm}$, cette couche se présente sous forme d'une coulée étalée sur plus de 3,5 mètres. Elle s'amincit dans sa partie Nord où elle repose directement sur la couche 17. Le sédiment est de structure laminaire peu développée. 
Couche 14 : d'épaisseur maximale $90 \mathrm{~cm}$, elle forme une masse de sédiment sablo-argilo-limoneux, compact, dur et de couleur jaune à brunâtre.

Couche 13: d'épaisseur maximale $15 \mathrm{~cm}$. Elle est composée de limons faiblement argilo-sableux et présente une structure laminaire.

Couche 12 : d'épaisseur maximale $7 \mathrm{~cm}$, elle est composée de sables quartzeux micacés renfermant quelques rares granules siliceux assez émoussés.

Couche 11: d'épaisseur maximale $20 \mathrm{~cm}$, elle est constituée d'un sédiment argilo-limoneux, disposé en minces lits assez réguliers et d'épaisseur 2 à $3 \mathrm{~cm}$, intercalés par des lamines de sables très fins.

Couche 10: d'épaisseur maximale $50 \mathrm{~cm}$, elle est composée essentiellement de sable siliceux et micacé avec de rares granules quartzeux.

Couche 9: d'épaisseur maximale $5 \mathrm{~cm}$, c'est un limon argileux, compact, de structure laminaire, peu dur et de couleur brun très pâle.

Couche 8: d'épaisseur maximale $15 \mathrm{~cm}$, elle est formée de sables siliceux micacés. Le sédiment est peu compact et de couleur brun très pâle.

Couche 7 : d'épaisseur maximale $1,20 \mathrm{~m}$, cette couche constitue une coulée importante de sédiment sur plus de 3 mètres de largeur. Elle recoupe en partie les couches 8 à 14 et forme ainsi une poche de 1,20 $\mathrm{m}$ de profondeur. Il est très compact. Cette couche renferme également des blocs calcaires de taille variable pouvant atteindre un mètre de dimension, ainsi que des fragments de plancher stalagmitique.

Ensemble supérieur : Dans cet ensemble, on distingue de bas en haut les unités stratigraphiques suivantes :

Couche 6: elle est visible sur une épaisseur variable pouvant atteindre 5 mètres. Les dépôts de cette couche sont composés de sables argilo-limoneux englobant quelques cailloutis calcaires de taille variable ainsi que des blocs d'effondrement de dimensions importantes.

Couche 5 : d'épaisseur maximale $55 \mathrm{~cm}$, elle est moins développée que la couche 6 . Elle perd de son importance en allant vers le Nord du profil pour disparaitre en biseau. Le sédiment de cette couche est de texture sabloargilo-limoneuse. De rares cailloutis calcaires sont rencontrés dans cette couche également.

Couche 4 : d'épaisseur maximale $1,20 \mathrm{~m}$, elle est formée de sables faiblement limono-argileux, chargés de cailloutis de tailles variables et de blocs calcaires de dimensions moyennes.

Couche 3: d'épaisseur maximale $40 \mathrm{~cm}$, c'est un dépôt riche en éléments calcaires emballés dans une matrice de texture voisine de celle de la couche 4. Le sédiment fin est peu compact.

Couche 2 : c'est un dépôt de cailloutis calcaires emballés dans une matrice de texture sablo-argileuse. Les cailloutis sont dans leur majorité concrétionnés. 
Couche 1 : elle forme une poche de 2,50 $\mathrm{m}$ de long et de 1,20 $\mathrm{m}$ de large. Cette couche entre en contact avec les couches 4 à 7 qui les a ravinés en partie au sud de ce profil. Le sédiment fin et de texture sablo-argileuse, faiblement limoneuse. Au sommet de ce profil, un plancher stalagmitique plus ou moins fragmenté vient coiffer cette couche.

\section{Etude sédimentologique \\ Profil 1, zone A}

\section{Diagramme stratigraphique global}

L'étude du diagramme stratigraphique global de cette zone permet de distinguer deux parties : La partie supérieure de la séquence correspondant aux couches 1 à 4 . Ces couches sont dépourvues de sédiments grossiers.

-La partie inférieure est marquée par des variations de texture témoignant des conditions de sédimentation différentes qui se manifestent dans la compétence de l'agent susceptible de transporter ces sédiments à l'intérieur de la grotte. En effet, la base de dépôt correspondant à la couche 10 est caractérisée par un sédiment de texture fine. La couche 9 se distingue très nettement des couches adjacentes par un enrichissement très marqué en éléments supérieurs à $2 \mathrm{~mm}(68 \%)$. La couche 8 présente une texture assez fine comparable à celle de la couche 10. Elle est dépourvue d'éléments grossiers de diamètre supérieur à $2 \mathrm{~mm}$. Les éléments grossiers de taille supérieure à $2 \mathrm{~mm}$ sont prépondérants dans la couche 7 . Au niveau de la couche 6, les éléments grossiers font à nouveau défaut, et la texture du sédiment rappelle celle de la couche 8 . Enfin, la couche 5 s'individualise par la réapparition du sédiment supérieur à $2 \mathrm{~mm}$, qui forme $38 \%$ de l'ensemble du sédiment.

\section{Morphoscopie des grains de quartz}

Les grains sub-anguleux luisants dominent largement dans tous les échantillons examinés. Leurs proportions les plus élevées s'enregistrent dans les couches à sédiments grossiers. Leur pourcentage oscille entre 74 et $86 \%$ dans la partie inférieure de cette séquence, formée par les couches 10 à 5 . Ensuite, on remarque une diminution progressive de cette catégorie en allant vers le sommet des dépôts pour ne représenter finalement que prés de $50 \%$ dans la couche 1. Les grains non usés sont présents dans tous les échantillons analysés. Leurs taux varient de 12 à $20 \%$ au maximum, alors que les émoussés luisants sont faiblement représentés, et ne dépassent jamais $5 \%$ de l'ensemble des grains quartzeux observés. Les sub-anguleux à peine mats sont peu nombreux au niveau des couches inférieures (couches 10 à 8) dans lesquelles ils représentent moins de $5 \%$. Ils disparaissent dans la couche 7 , pour réapparaitre à nouveau à partir de la couche 6 . Leur taux augmente ensuite jusqu'à la couche 1 pour atteindre environ $20 \%$ de cortège des grains 
de quartz examinés. Il faut noter également l'apparition des grains arrondis à peine mats dans les couches 4 à 1 .

\section{Profil 1, zone B}

\section{Diagramme stratigraphique global}

L'analyse granulométrique fait apparaitre des coupures bien nettes, séparant les différentes couches observées lors de la description des différentes unités stratigraphiques dans cette partie du remplissage. Les couches supérieures sont dépourvues d'éléments grossiers à l'exception de rares cailloutis que renferment le dépôt formant la couche A. La série débute alors par un dépôt de texture fine (couches $\mathrm{L}$ et $\mathrm{K}$ ), puis on passe brutalement à un sédiment grossier dans lequel les éléments grossiers constituent plus de $70 \%$ du sédiment provenant de la couche J. Les éléments supérieurs à $2 \mathrm{~mm}$ ne sont que faiblement représentés au niveau de la couche I (12 \%). Ils sont beaucoup plus abondants dans la couche $\mathrm{H}$ puisqu'ils forment prés de $50 \%$ du sédiment. Dans la couche $\mathrm{G}$, les éléments de diamètre supérieur à $2 \mathrm{~mm}$ font défaut. Ils réapparaissent à nouveau dans la couche $\mathrm{F}$ où ils entrent pour $29 \%$ dans la composition de l'ensemble du sédiment. Dans la couche $\mathrm{J}$, c'est la fraction grossière qui est la mieux développée et représente environ $33 \%$ de sédiment total. Elle est absente de la couche I, forme ensuite $11,7 \%$ du sédiment global dans la couche $\mathrm{H}$ et seulement $4,8 \%$ du sédiment de la couche F. Leur taux maximal s'enregistre dans la couche $\mathrm{H}$ où elles forment $38,7 \%$ du sédiment total. C'est la fraction fine qui est la mieux développée, bien que sa proportion ne dépasse jamais $27 \%$ de l'ensemble du sédiment.

\section{Morphoscopie des grains de quartz}

Le cortège des grains de quartz est dominé par la catégorie des grains sub-anguleux luisants qui forment 70 à $85 \%$ dans toutes les couches sauf dans les échantillons provenant des couches $\mathrm{A}$ et $\mathrm{B}$ ou leur pourcentage reste tout de même supérieur à $50 \%$. Les grains émoussés luisants sont faiblement représentés et ne dépassent guère $8 \%$ au maximum. Les grains non usés sont assez développés dans la couche A $(25 \%)$; ils ne constituent que 6 à $12 \%$ dans les autres couches. Les proportions des sub-anguleux à peine mats sont assez variables d'une couche à l'autre. Ils sont maximum dans les couches D, $\mathrm{B}$ et $\mathrm{A}$, où ils entrent pour moins de $10 \%$ dans la composition du cortège des grains de quartz. Les arrondis mats font leur apparition dans les couches supérieures de la séquence, mais leur proportion ne dépasse pas $7 \%$.

\section{Pétrographie des galets (fig. 8)}

Le quartz et les quartzites forment la composante essentielle des éléments grossiers, puisqu'ils constituent prés de $42 \%$ de l'ensemble des 
galets examinés. Ces galets sont très émoussés, Les roches granitiques sont bien représentées et les galets qui en dérivent forment $27 \%$ de l'ensemble des éléments supérieurs à $10 \mathrm{~mm}$. On trouve des galets très altérés bien que peu nombreux, associés à d'autres peu ou pas altérés. Quelques fragments de roches métamorphiques (schistes, micaschistes, gneiss) sont reconnaissables à l'intérieur de ces dépôts sous forme de petits galets très altérés. Leur proportion ne dépasse pas $5 \%$ et ils s'émiettent assez facilement par une simple pression entre les doigts. Les galets gréseux sont relativement abondants dans ces dépôts et forment $18 \%$ du total des galets. Ils sont faiblement altérés et généralement de forme sub-arrondie à arrondie. Les silex sont moins représentés, avec un pourcentage de $8 \%$. L'association d'éléments altérés et non altérés laisse supposer que ces matériaux sont hérités de terrasses plus ou moins anciennes par remaniement (Duplex et Guillien ; 1967).
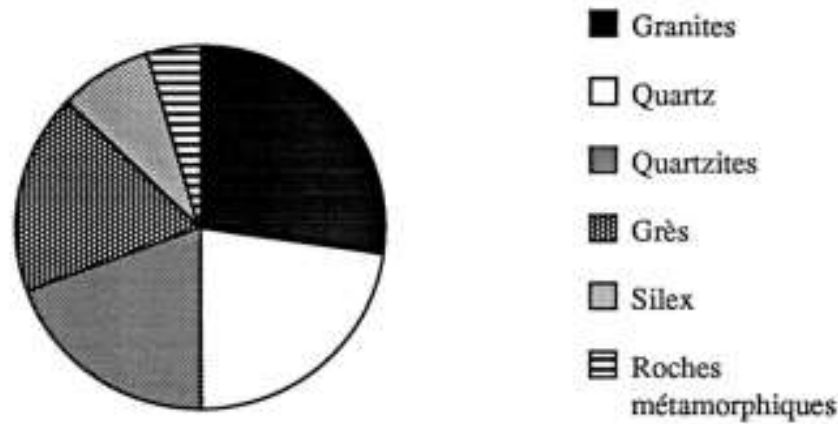

Fig. 8 : Diagramme circulaire de la pétrographie des galets, locus 3, profil 1.

\section{Profil 2}

\section{Diagramme stratigraphique global}

Le diagramme stratigraphique global montre une différence profonde entre la partie inférieure ou ensemble I, formée par les couches 26 à 7, et la partie supérieure ou ensemble II, qui regroupe les couches 6 à 1. Les variations texturales que met en évidence ce diagramme permettent de diviser cette partie du remplissage en deux grands ensembles sédimentaires Ensemble I : du point de vue granulométrique, cet ensemble peut être subdivise en deux sous-ensembles assez marques :

Sous-ensemble 1 : il comprend les couches 26 à 15 et se caractérise par l'alternance de couches à éléments grossiers (galets et graviers) d'une part, et $d^{\prime}$ 'autre part de couches à sédiments fins. Les éléments de diamètre supérieur à $2 \mathrm{~mm}$ représentent 30 à $60 \%$ du sédiment total. Leur taux le plus élevé est enregistre dans la couche 25 . Les éléments grossiers ne dépassent 
jamais 8,3\% du sédiment analysé sauf dans la couche 25 ou les galets constituent tout de même $27 \%$ de l'ensemble du sédiment.

Sous-ensemble 2 : il comprend les couches 14 à 7 et se distingue du sous-ensemble 1 par l'absence des éléments grossiers dont le diamètre dépasse $5 \mathrm{~mm}$. Il se caractérise également par l'alternance de couches à texture fine, limono-argileuse, et de couches à texture sableuse. Les granules forment prés de $18 \%$ du sédiment dans les couches 10 et 12 . Ils sont pratiquement absents dans les autres couches.

Ensemble II : l'étude granulométrique, révèle la nature peu grossière du sédiment provenant des couches 6, 5 et 4, puisque les éléments grossiers de diamètre supérieur à $2 \mathrm{~mm}$ ne dépassent guère $16 \%$ au maximum. En revanche, les couches 3 et 2 paraissent plus chargées en cailloutis calcaires qui forment 61 à $66 \%$ de l'ensemble du sédiment. Cet enrichissement en sédiment grossier s'accompagne d'une augmentation de la taille des éléments grossiers.

\section{Morphoscopie des grains de quartz}

Cet examen morphoscopique souligne l'abondance relative des grains sub-anguleux luisants. Leur pourcentage varie de 37 à $45 \%$. Les anguleux sont également assez nombreux et représentent 18 à $26 \%$ du cortège des grains de quartz analysés. Les arrondis mats sont présents dans tous les échantillons prélevés dans cet ensemble. Ils entrent pour 8 à $13 \%$ dans la composition du cortège de grains quartzeux. Les grains sub-anguleux à peine mats constituent 22 à $30 \%$. Enfin, les émoussés luisants sont très rares ou absents dans les couches 1 à 5. Ils forment moins de $2 \%$ au niveau de la couche 6 .

\section{Corrélations stratigraphiques entre les deux profils 1 et 2 du secteur II}

Pour le profil I, le rapport stratigraphique entre la zone A et la zone B se fait directement grâce a la continuité des dépôts composant d'un coté les couches 5, 4, 3 et 2 de la zone A, et de l'autre coté les couches F, E, D et B de la zone $\mathrm{B}$. La couche $\mathrm{C}$ n'apparait dans cette dernière zone que sous forme de lentille. En ce qui concerne la corrélation des dépôts de la partie inférieure, les observations effectuées sur le terrain et la comparaison des résultats sédimentologiques permettent d'établir une corrélation stratigraphique entre les différentes unités sédimentologiques de ce profil. Ainsi, les couches 10, 9, 8, 7et 6 décrites dans la zone A correspondent respectivement aux couches $\mathrm{K}, \mathrm{J}, \mathrm{I}, \mathrm{H}$ et $\mathrm{G}$ observées dans la zone B du même profil. Dans le cas du profil 2, le complexe de dépôts composant les unités stratigraphiques 20 à 15, décrites dans ce profil, sont héritées des couches qui figurent dans la zone B du profil 1, lors du glissement d'une partie de ces derniers dépôts autorisent à faire correspondre l'ensemble des 
couches $\mathrm{L}$ à $\mathrm{E}$ de la zone $\mathrm{B}$ du profil 1 aux dépôts regroupant les couches 20 à 15 décrites au niveau du profil 2. D'autre part, ils ont permis également de corréler la couche $\mathrm{D}$ de la zone $\mathrm{B}$ du profil 1 à la couche $14 \mathrm{du}$ profil 2 . A partir de ce niveau du remplissage, l'évolution des dépôts n'est pas la même dans les deux profils et la corrélation entre les autres couches reste difficile à établir. De même, les couches inférieures du profil 2 (couches 26 à 21) ne trouvent pas de dépôt équivalent dans le profil 1.

\section{Interprétations dynamique et climatique des dépôts Profil 1, secteur II}

Au niveau de ce profil, les dépôts à caractères fluviatiles témoignent certainement de conditions climatiques tempérées et humides. Les variations bien marquées de texture des sédiments sont liées directement aux fluctuations de la compétence des eaux ayant transporté et mis en place ces matériaux. Ainsi, les couches J, H et F de la zone B d'un coté et les couches 9,7 et 5 de l'autre coté représentent des épisodes à ruissellements intenses, susceptibles d'entrainer des galets pouvant atteindre environ $50 \mathrm{~mm}$ de diamètre. On remarque également que ces ruissellements perdent progressivement de leur énergie pendant ces mêmes épisodes au fur et à mesure que l'on s'éloigne de la base vers le sommet de cette séquence. Dans la zone A, la couche 10 a été fortement ravinée par les dépôts de la couche 9. $\mathrm{Au}$ niveau de la zone $\mathrm{B}$, les dépôts commencent également par un sédiment très fin, témoignant d'une mise en place par ruissellement diffus et de faible compétence. $\mathrm{Au}$ dessus de ces unités stratigraphiques, les sédiments des couches $\mathrm{J}$ à $\mathrm{E}$ sont bien homogènes et traduisent un tri franchement fluviatile. L'aspect "lavé" des sédiments dans les couches à texture grossière de ce profil et l'absence d'un granoclassement de ces dépôts témoignent d'une mise en place par excès de charge, pendant des périodes d'alluvionnement, suffisamment intenses pour entrainer les particules très fines. L'examen morphoscopique des grains de quartz confirme ce transport par l'eau, puisque la catégorie des grains de quartz luisant domine largement dans tous les échantillons examinés. L'association des grains de quartz peu usés à d'autres émoussés dans une même couche laisse penser à une reprise d'anciennes alluvions au cours de leur transport. La présence de certains grains arrondis à peine mats peut avoir plusieurs interprétations, mais l'absence de grains typiquement éoliens (ronds mats) laisse supposer une altération chimique assez modérée qui a du dépolir la surface des grains à peine mats.

\section{Profil 2, secteur 2}

Le phénomène majeur à signaler dans ce profil est le glissement

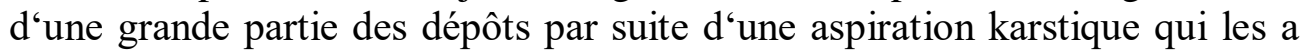


affecté et a provoqué un affaissement importante. En ce qui concerne le type de climat qui a présidé leur édification, les couches inférieures à caractères fluviatiles (ensemble I) correspondent à des conditions climatiques tempérées et humides. D'une manière générale, les variations de texture d'amplitude plus ou moins importante de ces dépôts sont liées essentiellement à l'intensité du ruissellement qui a transporté les sédiments et les a mis en place à l'intérieur de la grotte. Par conséquent, la grossièreté des matériaux de chaque couche est liée aux variations du degré de l'humidité qui leur est contemporaine. Dans le détail, les couches 25 et 23, chargées en éléments grossiers témoignent de ruissellements relativement intenses. En revanche, les ruissellements qui ont mis en place les couches 26, 24, 22 et 21 étaient de faible énergie. La couche 26 semble être ravinée en partie par les dépôts sus-jacents, ce qui traduit une variation brutale de la compétence du courant d'eau d'une couche à l'autre. L'ensemble des couches 13 à 8 témoigne d'épisodes suffisamment humides pour que les ruissellements aient pu entrainer et mettre en place des sédiments sableux, alternés avec d'autres épisodes relativement moins humides permettant le dépôt de sédiments très fins dans des eaux calmes. La structure finement laminaire des niveaux et couches à sédiments limono-argileux montre que ces dépôts ont été mis en place par décantation. Ces couches 13 à 8 ont été tronquées par la couche 7 glissée par éboulement à partir des niveaux supérieurs. Le sédiment composant cette dernière témoigne également d'un dépôt sous l'eau, comme l'indique sa sous-structure laminaire. Les microfissures qui ont affecté les niveaux à sédiment fin de cette partie du remplissage, notamment les couches 14 à 9, expliquent que ces dépôts étaient suffisamment secs lors de la mise en place de la couche 7 , ce qui a provoque ces cassures. L'hétérogénéité des sédiments fins provenant de ces couches montre que ces dépôts ont été remaniés vraisemblablement par solifluxion. L'effondrement des gros blocs qui figurent au sein de ce remplissage parait contemporain de l'édification de ces dépôts. La couche 4 moins chargée en cailloutis calcaires correspond à des conditions climatiques relativement peu froides et peu humides. Son sédiment fin en grande partie carbonaté résulte de la désagrégation et de l'émiettement de la roche encaissante. Les particules non calcaires que renferme le matériel fin sont apportées par un ruissellement de faible compétence. La présence des grains de quartz à aspect luisant d'une part, et d'autre part le mauvais classement du résidu non calcaire approuvent cette interprétation. Pendant la mise en place des couches 3 et 2, le climat devient nettement plus rigoureux, comme en témoigne le taux élevé des éléments calcaires grossiers. L'augmentation du pourcentage de la catégorie des grains quartzeux à aspect mats confirme cette aggravation des conditions climatiques. 
Essai chronostratigraphique $\mathrm{La}$ formation $\mathrm{du}$ plancher stalagmitique ainsi que les dépôts allochtones étudiés dans le locus 3 , profil 1 sont attribués soit au début du Würm ancien, soit à l'interglaciaire RissWürm (Debenath, 1974, 1976 ; Duport, 1971, 1976 ; Marquet, 1986). La puissance de ces dépôts à caractère fluviatile dont la base n'a pas été atteinte au niveau du profil 2 de ce locus, ainsi que l'importance du plancher stalagmitique montrent que cette période a du être assez longue et bien marquée.

\section{Conclusion}

L'étude sédimentologique des deux secteurs du grand porche ont permis d'établir un rapport stratigraphique entre les différents zones du secteur II. Ce rapport se fait notamment grâce à la continuité des sédiments qui compose les couches 5, 4, 3 et 2 de la zone A du profil 1 (secteur II) d'une part et les couches F, E, D et B de la zone B du même profil d'autre part. Au niveau du secteur I, les variations latérales matérialisées par les caractéristiques sédimentologiques nous ont permis de corréler la couche 2 $\mathrm{du}$ profil 1 avec la couche $4 \mathrm{du}$ profil 2. D'autre part, les analyses sédimentologiques ont montré que cette partie du remplissage sont composés surtout de sédiments allochtones, constitués essentiellement d'éléments de roches siliceuses. L'aspect luisant des grains de quartz confirme l'origine allochtone de ces dépôts. Ces dépôts témoignent alors d'un climat humide contemporain à leur mis en place. Les dépôts de toutes les couches sont formés de ce type de dépôts à l'exception de la couche 1 qui est composé d'éléments autochtones. L'étude des dépôts sédimentaires qui se sont accumulés avec le temps dans cette partie de la grotte ont permis de discerner dans ce remplissage les conditions tempérées et humides comme en témoigne la nature siliceuses des sédiments au niveau des couches $\mathrm{J}, \mathrm{H}$, et $\mathrm{F}$ du profil 1, zone B et les couches 9, 7 et 5 du profil 1, zone A. Les variations texturales des sédiments d'une couche à une autre reflètent les variations de l'intensité des ruissellements qui ont transportés et déposer ces sédiments. A partir de la couche 4, la présence des cailloutis calcaires d'origine autochtone témoigne d'un climat qui devient relativement froide. C'est au niveau des couches 3 et 2 du profil 2, secteur II que le climat devient nettement plus rigoureux comme en témoigne le taux très élevé des éléments grossiers calcaires provenant des parois de la roche calcaire encaissante.

\section{References:}

1. BOUKHIR M. (1992). Etude sédimentologique et stratigraphique du gisement de Montgaudier. Thèse de Doctorat d'Université, Bordeaux I. 
2. BOUKHIR M. (2017). Etude sédimentologique et stratigraphique des dépôts des abris Gaudry et Lartet du gisement de Montgaudier, Charente, France. European Scientific Journal February 2017 Edition vol.13, No.6

3. DEBENATH A. (1974). Recherches sur les terrains quaternaires charentais et les industries qui leur sont associés. Doctorat d'état es sciences, université de Bordeaux I, 1974.

4. DEBENATH A. 1976 - Les civilisations du paléolithique moyen en Charente. In «La préhistoire française », sous la direction de $\mathrm{H}$. de LUMELEY. CNRS éd., Paris, 1976, 2: 1070-1076.

5. DUPLEX S. et GUILLIEN Y. 1967 - Le plio-quaternaire de la Tardoire, Charente. Bull. A.F.E.Q., 1967, 2: 171-175.

6. DUPORT L. 1971 - Découverte de deux crânes humains dans la grotte de Montgaudier. C.R. Acad. Sc., Paris, 1971, 283: 1015-1016.

7. DUPORT L. 1976 - La mandibule moustérienne de Montgaudier, Charente. C.R. Acad. Sc., Paris 1976, 283: 1161-1164.

8. MARQUET J. C. 1987 - Contribution à la connaissance de la chronologie du site de Montgaudier d'après les rongeurs. In «Préhistoire de Poitou-Charentes, problèmes actuels », actes du $11^{1 \text { ème }}$ congrès national des sociétés savantes, Poitiers, 1986. Editions du CTHS, Paris, 1987, pp : 49-59. 